Anaesthesist $2010 \cdot 59: 86-88$

DOI 10.1007/s00101-009-1652-7

Online publiziert: 20. Januar 2010

๑) Springer-Verlag 2009

\title{
Neurologische Komplikation nach einer vertikalen infraklavikulären Plexusblockade
}

tierte Blockade zu liegen. Die am Operationsabend noch bestandene motorische Blockade $(7,5 \mathrm{~h}$ nach lege artis angelegter Plexusblockade) kann als normal betrachtet werden.

Die weiteren neurologischen Abklärungen im Verlauf bei persistierender $\mathrm{Pa}$ rese des rechten Arms und distalen Dysästhesien umfassen leider keine Elektromyographie- (EMG-)Untersuchung. (Zumindest werden in diesem Fallbericht keine erwähnt.) Die F-Welle (F-Antwort), eine physiologische motorische Spätantwort im EMG, die nach Reizung eines Nervs auftritt, kann bei Störungen einer generalisierten Beeinträchtigung der Nervenleitung (z. B. Polyneuropathie) oder bei proximalen längerstreckigen Prozessen, v. a. im Plexus- und Wurzelbereich, zu mehr Klarheit verhelfen.

Die im Halswirbelsäulencomputertomogramm (HWS-CT) gefundenen massiven degenerativen Veränderungen von $\mathrm{C}_{3}-\mathrm{C} 6$, die im Magnetresonanztomogramm (MRT) eine ventrale und dorsale Kompression des Myelons bei ausgedehntem Bandscheibenvorfall auf Höhe C5/C6 zum Vorschein brachten, könnten mit den klinischen Symptomen in diesem beschriebenen Fall und der durchgeführten Anästhesie (infraklavikuläre Plexusblockade) auch im Rahmen eines „double crush syndrome“ erklärt werden. Das Double crush syndrome wurde erstmals 1973 von Upton u. McComas [5] sowie 1988 Osterman [6] beschrieben. Es betrifft die Summationseffekte von Allgemeinerkrankungen und subklinischen Nervenkompressionssyndromen bzw. die Summation zweier Nervenkompres- sionen im Sinne axoplasmatischer Flussstörungen.

Im Übrigen soll erwähnt werden, dass ein persistierendes neurologisches Defizit nach korrekter Technik und Durchführung einer peripheren Nervenblockade bereits von Dullenkopf et al. [7] beschrieben wurde.

\section{Korrespondenzadresse \\ Prof. A. Borgeat \\ Anästhesiologie, Intensivmedizin, Uniklinik Balgrist \\ Forchstrasse 340, 8008 Zürich \\ Schweiz \\ alain.borgeat@balgrist.ch}

\section{Literatur}

1. Zetlaoui PJ, Labbe JP, Benhamou D (2008) Ultrasound guidance for axillary plexus block does not prevent intravascular injection. Anesthesiology 108(4):761

2. Loubert C, Williams SR, Helie F, Arcand G (2008) Complication during ultrasound-guided regional block: accidental intravascular injection of local anesthetic. Anesthesiology 108(4):759-760

3. Schafhalter-Zoppoth I, Zeitz ID, Gray AT (2004) Inadvertent femoral nerve impalement and intraneural injection visualized by ultrasound. Anesth Analg 99(2):620-628

4. Koscielniak-Nielsen ZJ, Rasmussen $\mathrm{H}$, Hesselbjerg L (2008) Pneumothorax after an ultrasound-guided lateral sagittal infraclavicular block. Acta Anaesth Scand 52(8):1176-1177

5. Upton AR, McComas AJ (1973) The double crush in nerve entrapment syndromes. Lancet 302(7825):359-362

6. Osterman AL (1988) Double crush syndrome. Orthop Clin North Am 19:147-155

7. Dullenkopf A, Zingg P, Curt A, Borgeat A (2002) Funktionsverlust der oberen Extremität nach Bankart-Schulteroperation unter InterscalenusBlockade und Allgemeinanästhesie. Anaesthesist 51(7):547-551 


\section{Erwiderung}

\section{R. Ehrenberg}

Universitätsklinik für Anästhesiologie und operative Intensivmedizin, Martin-LutherUniversität Halle/Wittenberg

Wir bedanken uns für die kritische Auseinandersetzung mit der Problematik unseres Fallberichts, müssen aber auch darauf hinweisen, dass die Probleme des klinischen Alltags ebenfalls widergespiegelt werden. Die Entscheidung für eine periphere Nervenblockade (PNB) bei bestehendem neurologischen Defizit unter Vollheparinisierung wurde intern bereits vor Durchführung und auch später in der Diskussion kritisch hinterfragt. Die von Risch et al. zitierten Fallberichte (Literaturquellen 1-4 im Leserbrief) behandeln mögliche Komplikationen und lassen keine allgemeingültige Aussage über eventuelle Risiken zu. Wir möchten das mögliche Risiko einer versehentlichen intraneuralen Injektion oder vaskulären Punktion auch in geübter Hand nicht verharm- losen. Die Inzidenz eines persistierenden neurologischen Schadens durch eine PNB ist jedoch als sehr gering anzusehen, unabhängig von der Verwendung von Ultraschall (US) oder Nervenstimulation (NS; [1]). Gerade durch die geringe Inzidenz ist es nahezu unmöglich, eine kontrollierte randomisierte Studie durchzuführen. Die benötigte Patientenzahl läge bei $>10.000$, eine Inzidenz von o,018 vorausgesetzt $[1,2]$. Dagegen wurde für die vaskuläre Punktion eine deutliche Reduktion durch die Anwendung von US beschrieben $[3,4]$.

Die Verwendung einer 0,75\%igen Ropivacainlösung entspricht dem aktuellen Standard unserer Abteilung und stellt keinen Versuch dar, die Blockadequalität zu verbessern. Über die Einführung einer 0,5\%igen Lösung wird aber diskutiert. Für eine US-assistierte Blockade liegt, zugegeben, die Injektionsmenge von $30 \mathrm{ml}$ $(225 \mathrm{mg})$ an der oberen Grenze, aber dennoch unterhalb der empfohlenen Maximaldosis (bezogen auf das Körpergewicht). Daten über die Pharmakokinetik von Ropivacain aus eigenen unveröffentlichten Untersuchungen und anderen Studien belegen aber um ein Vielfaches niedrigere Plasmaspiegel im Vergleich zu Fallberichten einer versehentlichen intravasalen Injektion auch geringer Mengen von Ropivacain [5, 6]. Als theoretischer Vorteil dieser Menge erschien uns aber bei der Durchführung eine effizientere epineurale Verteilung ohne mehrfache Nadelkorrektur. Primär wurde nur der posteriore Faszikel aufgesucht; auf zusätzliche selektive Blockaden der anderen Faszikel wurde aufgrund der Vollheparinisierung bewusst verzichtet.

Eine erweiterte apparative neurologische Diagnostik hätte mit Sicherheit schon präoperativ aufschlussreiche $\mathrm{Zu}$ satzinformationen über die Ursache des neurologischen Defizits liefern können, wurde aber wegen der Diagnose des akuten Gefäßverschlusses mit dringender Operationsindikation nicht durchgeführt.

Bezüglich der Dauer einer motorischen Blockade nach Applikation von

\section{Hier steht eine Anzeige.}

\section{Springer}


o,75\%igem Ropivacain stimmen wir den Ausführungen von Risch et al. zu. Die Reihenfolge der Diagnostik am ersten postoperativen Morgen bestand nach neurologischer Konsultation aber primär im Einsatz der bildgebenden Verfahren MRT und CCT. Die weitere Diagnostik hätte mit Sicherheit auch das von Risch et al. erwähnte EMG umfasst. Im Verlauf kam es aber zu einer spontanen Verbesserung der Symptomatik. Als Ursache des neurologischen Defizits wurde nun ein zervikaler Bandscheibenvorfall diagnostiziert. Im Verlauf lehnte der Patient leider weitere diagnostische Maßnahmen und die notwendige neurochirurgische Therapie ab.

Persistierende neurologische Defizite nach „korrekter“ Durchführung einer vertikalen infraklavikulären Plexusblockade sind nicht nur von Dullenkopf et al. beschieben worden; Quellen zu weiteren Fällen werden in seiner Arbeit zitiert. Dieses Risiko und der Nutzen der PNB sind nun die Kernpunkte unserer kritischen Auseinandersetzung. Wie von Brull et al. [7] in einer Metaanalyse zusammengefasst, ist die Datenlage über Art und Ursache von nervalen Störungen nach PNB insgesamt eher lückenhaft. Diese Daten werden hauptsächlich von den schon oben erwähnten 2 französischen Studien geliefert und beschreiben 20 neurologische Defizite bei ca. 65.00o PNB über einen Zeitraum von 3 Jahren (ca. 3:10.ooo; $[1,2])$. Dabei soll auch darauf hingewiesen werden, dass für interskalenäre Blockaden das höchste Risiko (2,84:10o) für neurologische Defizite, wie im Beitrag Dullenkopf et al., besteht.

Interessant erscheint uns der Ansatz, das postoperative neurologische Defizit mit dem von Upton u. McComas beschriebenen Double crush syndrome zu erklären. Diese Hypothese basiert aber nicht auf vorbestehenden Allgemeinerkrankungen, sondern beschreibt nur den Summationseffekt von proximalen und distalen Nervenläsionen. Unabhängig voneinander bilden sie klinisch ein Symptom. Sie sollte initial als eine mögliche Erklärung für „chirurgische Versager" nach Karpaltunnelspaltungen bei Patienten mit Halswirbelsäulenbeschwerden dienen und wurde schon kurz nach ihrer Veröffentlichung kritisiert sowie an- gezweifelt. Nach unserer Kenntnis existieren für diese Hypothese keine validen Untersuchungen [z. B. EMG, Erhebung somatosensorisch-evozierter Potenziale (SSEP)]. Dennoch würde diese Hypothese die fehlende postoperative Verbesserung der neurologischen Symptomatik erklären. In Anlehnung an die „Doublecrush-Hypothese“ würden wir den Gefäßverschluss als distale Läsion und den nichtdiagnostizierten Bandscheibenvorfall als proximale Nervenläsion betrachten. Die durchgeführte Plexusanästhesie führt in diesem Fall zu einer verspäteten postoperativen Demaskierung und stellt keine zusätzliche Nervenläsion dar. Wir sollten grade in solchen Situationen die eigene Fehlbarkeit nicht außer Acht lassen, da auch die „korrekte“ Durchführung einer PNB eine „Nervenläsion“ darstellt und den axonalen Plasmafluss beeinträchtigt. Als Fazit dieses Gedankenaustausches würden wir gern nochmals auf die zwingende Notwendigkeit hinweisen, PNB und deren Risiken nicht zu verharmlosen und möglichen Komplikationen bis zur endgültigen Diagnose entschlossen nachzugehen. Dieser Gedanke stellt auch den Kern unseres Fallberichts über mögliche Differenzialdiagnosen eines neurologischen Defizits nach PNB dar.

\section{Korrespondenzadresse \\ Dr. Ramin Ehrenberg}

Universitätsklinik für Anästhesiologie und operative Intensivmedizin

Martin-Luther-Universität Halle/Wittenberg

06120 Halle/Saale

Ramin.Ehrenberg@medizin.uni-halle.de

Interessenkonflikt. Die korrespondierenden Autoren geben an, dass kein Interessenkonflikt besteht.

\section{Literatur}

1. Auroy Y, Narchi P, Messiah A (1997) Serious complications related to regional anesthesia: results of a prospective survey in France. Anesthesiology 87:479-486

2. Auroy Y, Benhamou D, Bargues I (2002) Major complications of regional anesthesia in France. The SOS Regional Anesthesia Hotline Service. Anesthesiology 97:1274-1280

3. Sauter AR, Dodgson MS, Stubhaug A et al (2008) Electrical nerve stimulation or ultrasound guidance for lateral sagittal infraclavicular blocks: a randomized controlled observer-blinded, comparative study. Anesth Analg 106:1910-1905
4. Abrahams MS, Aziz MF, Fu RF, Horn JL (2009) UItrasound guidance compared with electrical neurostimulation for peripheral nerve block: a systematic review and meta-analysis of randomized controlled trials. Br J Anaesth 102(3):408-417

5. Rettig HC, Lerou JG, Gielen MJ et al (2007) The pharmacokinetics of ropivacaine after four different techniques of brachial plexus blockade. Anesthesia 62:1008-1014

6. Satsumae T, Tanaka M, Saito S, Inomata S (2008) Convulsions after ropivacaine $300 \mathrm{mg}$ for brachial plexus block. Br J Anaesth 101(6):860-862

7. Brull R, McCartney CJ, Chan VW, El-Beheiry H (2007) Neurological complications after regional anesthesia: contemporary estimates of risk. Anesth Analg 104(4):965-974 